\title{
Research agenda on persistent and unpredictable threat of influenza and emerging respiratory infections: a public health necessity in the Eastern Mediterranean Region
}

\author{
Ahmed Mandil', Joseph Bresee ${ }^{2}$, Mohamed Awad Tageldin ${ }^{3}$, Talat Mokhtari Azad $^{4}$ and Wasiq Khan $^{1}$
}

Infectious diseases continue to represent a significant threat to global health security, particularly in the context of increasing globalization, interconnectedness and interdependence. Chief among such threats are influenza viruses and other respiratory pathogens, such as Middle East Respiratory Syndrome coronavirus (MERS-CoV), because of their risk of high transmissibility and acuity of illness. Annual epidemics of seasonal influenza cause an estimated 3-5 million cases of severe illness and more than 500000 deaths (1), with the prospect of pandemic influenza viruses causing far greater impact. In addition, the appearance of severe acute respiratory syndrome (SARS) in 2003, widespread and continued outbreaks of avian influenza A (H5N1) since 2004, the $\mathrm{H} 1 \mathrm{~N} 1$ pandemic in 2009 and emergence of MERS CoV in 2012 reflect the seriousness of public health challenges posed by influenza and emerging respiratory infections.

In 2009, WHO launched an ambitious public health research agenda for influenza that aimed to enhance the influenza evidence base (2). This was especially important since disease impact, best preventive and control approaches for influenza, and emerging respiratory infections are not well documented in the WHO Eastern Mediterranean Region (EMR). Recent literature reviews conducted by the WHO Eastern Mediterranean Regional Office (WHO/
EMRO) recognized an increase in published evidence on influenza, MERS $\mathrm{CoV}$ and acute respiratory diseases (ARD) with epidemic and pandemic potential. However, the reviews also acknowledged inconsistencies with the global research agenda for influenza, with progress being intermittent and delayed (WHO/EMRO, unpublished data).

The Pandemic Influenza Preparedness (PIP) Framework - an innovative global public/private partnership - was agreed by the World Health Assembly in 2011 (3). The Framework is considered an opportunity to enhance global efforts for pandemic preparedness and to promote research on influenza and novel respiratory pathogens that can cause the next pandemic. The World Bank estimates a future influenza pandemic could cost the world economy a staggering US\$3 trillion (4). Researching and building the evidence base for prevention and control of influenza and emerging respiratory infections may offer a cost effective alternative to the potential impact of an influenza pandemic or widespread epidemic of a novel respiratory disease. The Ebola virus disease (EVD) epidemic in Africa from 2014 to 2015, while not a respiratory disease, serves as an example of the impact of a major infectious disease outbreak in modern times. Moreover, it provides a number of lessons for the public health community, including the importance of investing in research for better preparedness (5).

The EMR is a region facing complex security and humanitarian situations, and investing time and effort in influenza and other respiratory diseases research might not appear to be a priority on its public health agenda (for the time being). However, there are several reasons why this investment is a necessity:

- The Region holds a central location for global migratory bird flyways (Central Asia-India, West Asia-Africa, Mediterranean-Black Sea, and East Atlantic). Aquatic birds are the reservoirs of influenza viruses that could infect humans and become the next pandemic strain (6). Indeed, the introduction of avian influenza A (H5N1) in Egypt and continuing sporadic human infections with the virus highlight the risk of avian influenza viruses in the Region.

- Influenza viruses are unpredictable, and as the emergence of the 2009 pandemic in the Americas indicated, it is not possible to say precisely when and where the next influenza pandemic will strike.

- Research capacity and research output in the Region are inadequate, and strengthening these will have long lasting benefits beyond influenza.

- The Region hosts annual religious gatherings of millions of people from across the globe (Hajj, Umrah, 
Mazarat) that provide perfect opportunities for transmission of novel respiratory and infectious pathogens. The emergence of MERS CoV and continued cases are a reminder of the risk of novel respiratory pathogens anywhere in the world and the resulting disease and public impact.

- Epidemic and preparedness is a complex process (7), demanding resources and political commitment, which benefit from a research agenda that engenders efficient investments. This complex set of reasons justifies promoting research on influenza and emerging respiratory infections in this Region.
This theme issue of the Eastern Mediterranean Health Journal (EMHJ) is a step forward in the promotion of public health research on influenza and emerging respiratory diseases in the Region. It includes a variety of contributions in the form of original research papers, reports, literature reviews and commentaries from diverse countries in the Region (including Afghanistan, Egypt, Islamic Republic of Iran, Jordan, Lebanon, Morocco, Saudi Arabia and Tunisia) based upon epidemiological (population-based modeling), clinical and laboratory-based studies. Inspired by the global push of the PIP Framework, this issue is expected to generate interest in emerging knowledge from the Region, which has witnesses more global attention on its conflicts than health issues. It is also an opportunity to stimulate debate on the importance of influenza, emerging respiratory diseases research in the Region and identify avenues for further enquiry. Although the central effort behind this theme issue of EMHJ was led by WHO/EMRO, its publication is still a reflection of the interest and untapped opportunities in the form of researchers from the Region who were ready to contribute to the world of science as well as public health, if given an opportunity and avenue to do so.

\section{References}

1. World Health Organization (2014). (http://www.who.int/mediacentre/factsheets/fs211/en).

2. World health Organization (2009). (http://www.who.int/ influenza/resources/research/en).

3. World Health Organization (n.d.) (http://www.who.int/influenza/pip/en).

4. The World Bank (2015). (http://www.worldbank.org/en/ topic/pandemics/overview).

5. Jacobsen KH, Aguirre AA, Bailey CL, Baranova AV, Crooks AT, Croitoru A, Delamater PL, Gupta J, Kehn-Hall K, Narayanan A,
Pierobon M. Lessons from the Ebola outbreak: action items for emerging infectious disease preparedness and response. EcoHealth 2016, 13(1):200-212.

6. Causey D, Edwards SV. Ecology of avian influenza virus in birds. J Infec Dis. 2008, 197(Supplement 1):S29-S33.

7. Knobler SL, Mack A, Mahmoud A, Lemon SM (eds.) The threat of pandemic influenza: are we ready? workshop summary 2005. National Academies Press. 